\title{
BMJ Open Identifying factors influencing sustainability of innovations in cancer survivorship care: a qualitative study
}

\author{
Robin Urquhart (D) , ${ }^{1,2,3,4}$ Cynthia Kendell, ${ }^{4}$ Evelyn Cornelissen, ${ }^{5}$ Byron J Powell, ${ }^{6}$ \\ Laura L Madden, ${ }^{2}$ Glenn Kissmann, ${ }^{7}$ Sarah A Richmond (iD , ,,8 \\ Jacqueline L Bender ${ }^{3,9}$
}

To cite: Urquhart $\mathrm{R}$,

Kendell C, Cornelissen E, et al. Identifying factors influencing sustainability of innovations in cancer survivorship care: a qualitative study. BMJ Open 2021;11:e042503. doi:10.1136/ bmjopen-2020-042503

- Prepublication history and additional material for this paper is available online. To view these files, please visit the journal online (http://dx.doi.org/10. 1136/bmjopen-2020-042503).

Received 07 July 2020 Revised 14 January 2021 Accepted 18 January 2021

Check for updates

(C) Author(s) (or their employer(s)) 2021. Re-use permitted under CC BY-NC. No commercial re-use. See rights and permissions. Published by BMJ.

For numbered affiliations see end of article.

Correspondence to Dr Robin Urquhart: robin.urquhart@nshealth.ca

\section{ABSTRACT}

Objectives Moving innovations into healthcare organisations to increase positive health outcomes remains a significant challenge. Even when knowledge and tools are adopted, they often fail to become integrated into the long-term routines of organisations. The objective of this study was to identify factors and processes influencing the sustainability of innovations in cancer survivorship care.

Design Qualitative study using semistructured, in-depth interviews, informed by grounded theory. Data were collected and analysed concurrently using constant comparative analysis.

Setting 25 cancer survivorship innovations based in six Canadian provinces.

Participants Twenty-seven implementation leaders and relevant staff from across Canada involved in the implementation of innovations in cancer survivorship. Results The findings were categorised according to determinants, processes and implementation outcomes, and whether a factor was necessary to sustainability, or important but not necessary. Seven determinants, six processes and three implementation outcomes were perceived to influence sustainability. The necessary determinants were (1) management support; (2) organisational and system-level priorities; and (3) key people and expertise. Necessary processes were (4) innovation adaptation; (5) stakeholder engagement; and (6) ongoing education and training. The only necessary implementation outcome was (7) widespread staff and organisational buy-in for the innovation.

Conclusions Factors influencing the sustainability of cancer survivorship innovations exist across multiple levels of the health system and are often interdependent. Study findings may be used by implementation teams to plan for sustainability from the beginning of innovation adoption initiatives.

\section{INTRODUCTION}

Across Canada, many studies have identified gaps in the delivery of cancer care whereby the care patients receive is not consistent with scientific evidence. ${ }^{1-8}$ The Canadian Strategy for Cancer Control estimated that cancer outcomes could improve by as much as $30 \%$ by routinely applying existing evidence in

\section{Strengths and limitations of this study}

- The sustainability of evidence-based innovations has been described as 'one of the least understood and most vexing issues for implementation research' and we provide a comprehensive inquiry of factors and processes influencing the sustainability of innovations in healthcare.

- We interviewed 27 implementation leaders and relevant staff from 25 cancer survivorship innovations that were implemented across Canadian jurisdictions.

- We used several implementation science frameworks and taxonomies to design our study and analyse and interpret the findings.

- This study focused solely on innovations in cancer survivorship, which may limit transferability to innovations in other areas of care, although there is no inherent reason why innovations in cancer survivorship should differ from innovations in other areas of chronic disease management.

- This is a critical area of inquiry, given there are limited empirical data on the processes by which innovations are sustained in clinical settings as well as the considerable investment over the past decade to implement beneficial innovations so more people have access to high-quality healthcare.

practice. ${ }^{9}$ Similarly, the WHO estimated that worldwide, one-third of cancer cases could be prevented and another one-third cured if evidence-based practices were consistently implemented and sustained in care. ${ }^{10}$ Importantly, even when knowledge and tools are put into practice, they often fail to become integrated into the long-term routines of organisations. ${ }^{11-13}$ This poor sustainment means many patients do not benefit from the best care possible. ${ }^{14-16}$

The sustainability of evidence-based innovations has been described as "one of the least understood and most vexing issues for implementation research'. ${ }^{17}$ In the past decade, a number of researchers have published 
conceptual models on innovation sustainability in healthcare, ${ }^{18-21}$ while recent reviews have provided syntheses of how researchers in the field define and approach sustainability. ${ }^{22}{ }^{23}$ Nevertheless, there remain limited empirical data on the processes by which innovations are sustained in clinical settings and the factors that influence sustainability. ${ }^{1724-26}$

Focusing on cancer survivorship care, this study examined whether and how various evidence-based innovations have been sustained. Cancer survivorship was the focus of this study for three reasons: (1) the number of cancer survivors has grown substantially due to advances in early diagnosis and treatment ${ }^{27}$; (2) cancer survivorship has become a strategic policy focus, with Canadian decisionmakers seeking ways to deliver care and implement innovations that address the needs and circumstances of this growing population; and (3) cancer research funders in Canada have explicitly stated a need to integrate what we know into survivorship programmes and policy. ${ }^{28}$ The specific objectives were to (1) identify factors influencing sustainability and (2) explore the processes that facilitate the sustainability of innovations in cancer survivorship care.

\section{MATERIALS AND METHODS \\ Study design}

We conducted a qualitative study, informed by the principles of grounded theory, ${ }^{29}$ on the sustainability of evidence-based innovations in cancer survivorship care that have been implemented across Canadian jurisdictions. An innovation was defined as new knowledge, tools or interventions (including programmes and services) that organisations are using for the first time. ${ }^{30}$ An innovation was considered evidence based if at least one published peer-reviewed study, using an experimental or quasiexperimental design, demonstrated improved outcomes for the target population. This is the criterion used by the National Cancer Institute for Research-Tested Intervention Programs specifically for cancer control and cancer survivorship interventions. ${ }^{31}$ Sustainability was defined as the continuation of the innovation's activities or outcomes beyond the initial implementation stage or initial funding period. ${ }^{32}$

\section{Patient and public involvement}

No patient involved.

\section{Conceptual frameworks}

The study was informed by Scheirer's work on sustainability, ${ }^{32}$ the dynamic sustainability framework (DSF) ${ }^{19}$ and Nilsen's taxonomy of implementation frameworks. ${ }^{34}$ Scheirer's work was used during sampling, specifically to identify innovations based on innovation type: those implemented by individual providers; those requiring coordination among multiple staff; new policies, procedures and technologies; capacity or infrastructure building; collaborative partnerships or coalitions; and broad-scale system change. The DSF proposes that the 'fit' between the innovation (specifically, interventions) and the setting is key to sustainability, and focuses on three main elements: the intervention, practice setting or context, and broader ecological system. This framework informed development of the interview guide (eg, questions and probes around the innovation, practice setting and broader healthcare system) and ongoing analyses/ interpretation. Nilsen's taxonomy was used during data analysis only to categorise the resultant findings (see below).

\section{Participants}

Participants were implementation leaders and relevant staff from across Canada involved in the implementation of a range of innovations in cancer survivorship care (eg, self-management tools, physical activity programmes and models of follow-up care). Recruitment involved a twophased process. First, we had to identify innovations of interest and, second, recruit leaders and staff involved in those innovations. The identification of innovations was multipronged: (1) viewing of all archived rounds and reviewing of all publications posted on the Canadian Cancer Survivorship Research Consortium website; (2) multiple PubMed searches with combinations of relevant search terms (eg, cancer, survivor*, Canada, rehabilitation, interventions, physical activity); and (3) speaking with the individual responsible for survivorship care and programming at all provincial cancer agencies (or their equivalent) to identify additional relevant initiatives in each province. On a final list of all potential innovations, we assessed whether each innovation was evidence based, as per the criterion described above. ${ }^{31}$

From those innovations deemed evidence based, we purposively recruited participants to maximise variation in cancer site, type of innovation ${ }^{33}$ and geographic setting. Individual recruitment involved purposive sampling to identify the implementation leader(s) and/or staff member(s) who was most directly involved in the implementation and/or sustainment of the innovation. These individuals were contacted by the lead author (RU) via email and invited to anticipate. Data collection continued until thematic saturation was reached. ${ }^{35}$

\section{Data collection}

We conducted one-on-one, semistructured telephone interviews with participants. An interview guide was developed based on the study objectives and the DSF using practical guidance from Patton ${ }^{36}$ and Rubin and Rubin. ${ }^{37}$ The interview guide is provided as an online supplemental file. The interviews focused on eliciting participants' understandings of the innovation, the process by which it was implemented, whether and how the innovation is sustained and the multilevel factors affecting its sustained use and impact. One master's trained research associate with experience in qualitative methods (LLM) conducted all interviews, which lasted approximately $40-60 \mathrm{~min}$. The interviewer had no prior relationship with any of the 
participants, and no repeat interviews were conducted. Field notes were taken during interviews to record interviewer observations and perceptions. All interviews were audiotaped and transcribed verbatim.

\section{Data analysis}

Consistent with grounded theory, the interview data were collected and analysed concurrently. An inductive approach, using constant comparative analysis, was used to analyse the interview transcripts. ${ }^{29}$ Analysis involved coding, constant comparison, and identification, organisation and refinement of categories. To help ensure consistency and conceptual clarity throughout the process of coding and categorisation, a coding framework (ie, 'codebook') was developed by the lead author (RU) and research associate (LLM). This was achieved through review of three transcripts and a team discussion. Next, the research associate used the codebook to code the remaining transcripts, with regular meetings between the same two individuals to review coding and the consistency of applying the codes to the interview text, consult with field notes and refine the codebook as needed. Qualitative software (NVivo V.10, QSR International, 2012) was used for data management and to facilitate comparison and synthesis of codes. Several full team meetings were also conducted to review coding and discuss emerging findings.

During a final 2-day team meeting, the resultant findings were categorised according to determinants, processes and implementation outcomes, and whether the data suggested a factor was necessary to sustainability or important but not necessary. Drawing on Nilsen's taxonomy of implementation frameworks, ${ }^{34}$ we categorised factors as those that help us understand and/or explain what influences outcomes (determinants), those that describe the processes that help translate innovations into practice (processes) and those that identify important aspects by which to evaluate the initial implementation (implementation outcomes). Regarding the latter, implementation outcomes were specifically defined as the effects of deliberate and purposive actions to implement new treatments, practices and services'. ${ }^{38}$ Determining whether a factor was 'necessary' or 'important but not necessary' was an iterative process that involved analysing participant perspectives on this issue as well as the data on whether and the extent to which a specific innovation was sustained (ie, continued activities or outcomes beyond the initial implementation stage or initial funding period, ${ }^{32}$ as described above) in the presence or absence of all resultant factors. If innovations were sustained in the absence of a particular factor, then this factor was deemed important but not necessary.

\section{RESULTS}

Twenty-seven participants from 25 unique cancer survivorship innovations based in six Canadian provinces (British Columbia, Alberta, Manitoba, Ontario, Quebec and Nova

\begin{tabular}{|c|c|}
\hline Characteristic & $\mathrm{n}\left(\%^{*}\right)$ \\
\hline \multicolumn{2}{|l|}{ Sex } \\
\hline Male & $5(18.5)$ \\
\hline Female & $22(81.5)$ \\
\hline \multicolumn{2}{|l|}{ Jurisdiction† } \\
\hline British Columbia & $4(14.8)$ \\
\hline Alberta & $4(14.8)$ \\
\hline Manitoba & $1(3.7)$ \\
\hline Ontario & $14(51.8)$ \\
\hline Quebec & $3(11.1)$ \\
\hline Nova Scotia & $1(3.7)$ \\
\hline \multicolumn{2}{|l|}{ Professional role } \\
\hline Researcher & $11(40.7)$ \\
\hline Clinician & $11(40.7)$ \\
\hline Decision-maker & $5(18.5)$ \\
\hline \multicolumn{2}{|l|}{ Years in role } \\
\hline$<10$ & $6(22.2)$ \\
\hline $10+$ & $21(77.8)$ \\
\hline
\end{tabular}

*Percentages may not add to $100 \%$ due to rounding. †Indicates place of employment of participant; some innovations were pan-Canadian in nature but hosted/led by a programme in a specific jurisdiction.

Scotia) participated in this study. Table 1 presents participant characteristics. All interviews took place from August 2017 to March 2018. Of the 25 innovations, 20 were sustained to some degree in that activities continued after the initial funding period. Five were not sustained. The innovations were grouped into five categories, depending on its intended purpose: physical activity programmes, psychological support/counselling, transition to survivorship programmes, transition to primary care programmes and return to life and lifestyle programmes. Eighteen were delivered in person, four were delivered online and three were delivered both in person and online.

Sixteen factors were perceived to influence sustainability: seven determinants, five processes, and four implementation outcomes (figure 1). Seven of these were deemed necessary while nine were important but not necessary. Table 2 presents all 16 factors with brief descriptions. The necessary determinants, discussed in detail below, were (1) management support; (2) organisational and system-level priorities; and (3) key people and expertise. Necessary processes were (4) adaptation; (5) stakeholder engagement; and (6) ongoing education and training. The only necessary implementation outcome was (7) staff and organisational buy-in for the innovation.

\section{Necessary determinants}

Management support

Participants continually voiced their experience that the support of middle and senior managers is imperative to 


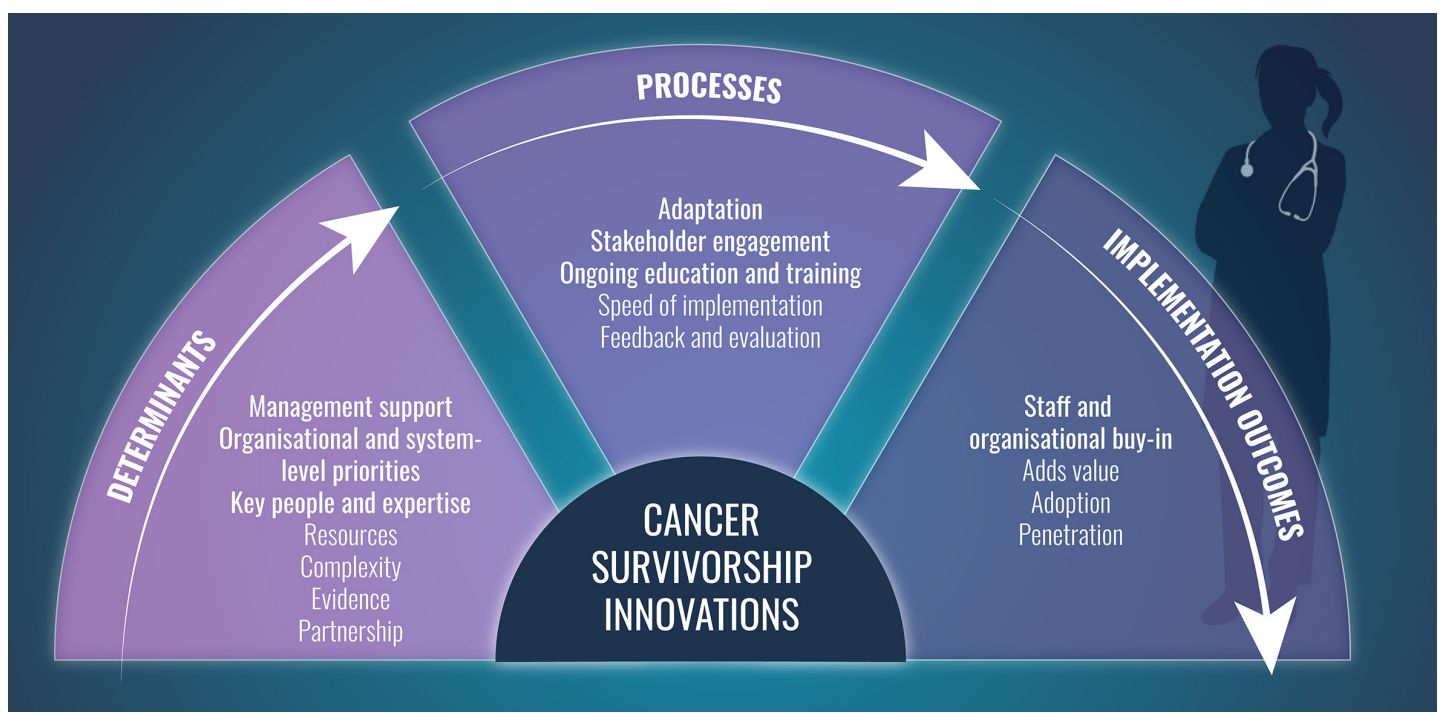

Figure 1 Factors influencing the sustainability of cancer survivorship innovations.

the sustained use of any innovation. Their experience was that even with all other pieces in place, it is extremely challenging to sustain any innovation without management support. As one participated stated, 'Management support, for sure, is very important, especially for growth. Um, very, very important' (Participant 19). Participants noted that management support tends to result in ongoing funding, whether this is the direct provision of funds (eg, out of their programme budget) or advocating for funding from other sources. Participants also described how it is often difficult for managers to support innovations in survivorship care because of competing priorities and that survivorship care does not result in quantifiable metrics in the same way other areas of care do:

I would say that it's one of the ... tougher components for people, for senior management, to buy into because it's a softer metric to try to collect in a way. Because it's not like you've got numbers of patients going through chemo or radiation. It's not, you know, survivorship care is a lot harder to look at that data and try to figure out if it's meaningful or worth it. (Participant 2)

Participants also noted that management support is much higher when an innovation and its sustainment are appropriately resourced and funded. Innovations that do not have secure funding require managers to transfer operational funds and/or allocate other resources (eg, staff time) away from existing programmes and services.

\section{Organisational and system-level priorities}

All participants discussed how survivorship care is perceived as a low organisational and health system priority relative to other cancer programmes and services. As one participant stated, 'It's not because people aren't interested in [survivorship care], it's just that it's maybe seen, maybe viewed as the nice to have, not the need to have' (Participant 2). As a result, the sustainment of innovations that have been implemented was described as particularly challenging, regardless of the extent to which programme components are in place and working well. Participants described several instances whereby survivorship care was prioritised and therefore initial implementation efforts were well supported and resourced. One example of this is a focus on postcancer treatment transitions mandated by the province of Ontario: 'Having Cancer Care Ontario ... starting to really implement comprehensive care that includes the patient, not just the tumour, is really helpful. Because our hospitals are funded by the degree to which they meet these mandates' (Participant 18). However, participants noted that even when innovations appeared to be integrated, shifting priorities at the health authority or government level often meant that sustainability was threatened. Speaking about an innovation related to transitioning survivors from active treatment to well follow-up care, one participant explained:

There had been significant shifting in terms of how our organization was structured and who actually had the authority and power, and their viewpoint on all of it. And, so, we couldn't at that point really proceed with it because the organization was really shifting away from that work. (Participant 11)

\section{Key people and expertise}

Participants continually emphasised the importance of two key individuals for ongoing sustainment of innovations: clinical champions and dedicated coordinators (or similar personnel). Ongoing champions were deemed necessary for sustainability. Several innovations illustrated this perspective. One was a programme aimed at transitioning low-risk survivors back to primary care after treatment. This programme, led by a tremendously well-respected clinical champion, had been in place for more than 4 years and appeared well integrated within the cancer care setting. However, on loss of the champion, the programme was substantially 
Table 2 Factors influencing the sustainability of cancer survivorship innovations

Factor Description

Determinants

Management support

The support of middle and senior managers is critical for sustainability. It is difficult to sustain any innovation in the absence of management support.

Organisational and system-level priorities

Survivorship care is generally not an organisational or system-level priority, making sustainability challenging. Even when survivorship is prioritised, shifting priorities at health authority or government levels often mean sustainability is threatened.

Key people and expertise

Key people, namely clinical champions and project/programme coordinators, are particularly important to maintaining an innovation's activities and use. Often, certain expertise or skill sets are required for an innovation to work efficiently and effectively.

Resources Resources in the form of funding, physical space and equipment are often very important to sustainment, particularly to expand a programme or service beyond the population served in the initial pilot phase.

Complexity Innovations that are simple, require less time to use, and the coordination and/or cooperation of fewer organisational members are easier to sustain.

Evidence Scientific evidence of an innovation's effectiveness contributes to sustainability by strengthening the case for funding, increasing its priority level and strengthening buy-in from front-line staff (mainly physicians).

Partnerships

Partnerships with other similar organisations, including community-based organisations, are not necessary for sustainability but can be very important as they permit the sharing of resources and expertise.

Processes

Adaptation

Adaptation, not fidelity, is necessary for sustainability. Adaptation is necessary to continually meet the needs of patients and to maintain fit with the local setting.

Stakeholder engagement The engagement of key stakeholders (eg, physicians, patients, administrators) is essential to sustainability by developing a sense of ownership over the innovation and allowing for practice-based adaptations that optimise fit with the local setting.

Ongoing education and training

Ongoing education and training is necessary to sustain innovations, particularly due to high levels of staff turnover in cancer care settings.

Speed of implementation

The speed of implementation can impact sustainability. Specifically, implementing slowly permits the time to get many of the key elements in place (eg, training and ongoing supports, metrics and data collection/reporting procedures, stakeholder engagement) that support the long-term sustainment of the innovation.

Feedback and evaluation

Feedback and evaluation, while not necessary, is important to sustainability as it helps to demonstrate the innovation's value, maintain credibility, maintain buy-in and help secure ongoing resources, including funding.

Implementation outcomes

Staff and organisational buy-in

Widespread and ongoing staff and organisational buy-in is necessary for sustainability. Many factors during the implementation period lead to buy-in.

Adds value

Adding value to the organisation (eg, through positive publicity) and its staff (eg, saving staff time) helps maintain buy-in, and increases opportunities for partnerships and additional resources.

Adoption

A lack of adoption, specifically by patients, threatens sustainability. Many survivorship innovations rely on patients being aware that a particular programme or resource is available and choosing to access it. Low patient uptake reinforces the perception such innovations are low priority.

Penetration Integrating the innovation into the service setting and its existing subsystems is important to sustainability. These systems include existing clinical workflows, including EMRs, physician ordering and other forms of documentation. Such integration can provide automatic referrals for programmes/services and serve as reminders regarding use.

Bolding represents necessary factors.

EMR, electronic medical records.

altered and eventually dwindled to minimal use. Conversely, two other transition programmes, in other jurisdictions, did not secure ongoing funding after the initial pilots, but maintained ongoing activities simply because the clinical champions continued the service, sometimes in a voluntary role (ie, during evenings 
and weekends) despite a lack of funding and other resources.

For many programmes, participants also described dedicated programme coordinators (or staff members with a coordination role) as being a necessary resource for sustainability, playing a complementary role to clinical champions. Their experience was that such a role was necessary to ensure the innovation was running smoothly, including the continuation of activities and tracking of deliverables. As one participant said:

It could conceivably be just a small team or one person working remotely, coordinating this kind of thing and sort of, like I said, overseeing the [innovation] and making sure that technological and referral purpose is running smoothly. But, it has to be, it does have to be there. It can't run itself. (Participant 7)

Many participants highlighted that certain expertise or skill sets are often needed for an innovation to continue to work efficiently and effectively. An example was the presence of certified exercise professionals for physical activity programmes.

\section{Necessary processes}

Adaptation

All participants emphasised that adaptation, not fidelity, is necessary for sustainability. Participants discussed how their task, as leaders and staff of innovations, was to ensure that the innovation evolved and adapted as necessary in their particular setting. Their view was that without adaptation, there was no sustainability. Adaptation was necessary to allow the team/organisation to continually meet the needs of patients and to maintain fit with the setting/ environment (eg, changing staff, capacity, resources, policies and political environment). As one participant stated:

Our being flexible and adapting to what would work, both for ourselves and our limited resources and time and for our patients. I think if we hadn't adapted the program, we wouldn't still be offering it. So, we had to adapt and change and shorten and condense, while sticking to the hearts and, you know, key concepts of the program. (Participant 17)

Table 3 provides examples of adaptations to each type of innovation. Many were related to delivery mechanisms with the goal of increasing accessibility of the programme and/or its feasibility (eg, changing the frequency or timing of delivery, moving some components to online delivery, changing referral processes). Moreover, it was widely recognised that adaptation was necessary because the evidence base for innovations changed. An innovation today, both its components and target population, will likely change as new evidence becomes available: 'I think it's imperative to keep current with the evidence for whatever it is you're offering. And making adaptations with the program that are in keeping with the evidence' (Participant 6).

\section{Stakeholder engagement}

Participants described the engagement of important stakeholders (eg, physicians, patients, administrators) as essential to sustainability. The data indicated participants viewed engagement as critical for two reasons. First, engagement increases awareness of the innovation, its evidence base and its potential benefits, and helps to

Table 3 Innovation types and examples of adaptations

\begin{tabular}{lll}
\hline Innovation type & Function & Examples of adaptations \\
\hline Physical activity programmes & $\begin{array}{l}\text { To increase physical activity among } \\
\text { cancer survivors }\end{array}$ & $\begin{array}{l}\text { Changes in timing and length of delivery; } \\
\text { changes in setting (cancer centre vs } \\
\text { community) }\end{array}$ \\
Psychological support/counselling & $\begin{array}{l}\text { To provide cancer survivors with } \\
\text { the tools to manage/cope with } \\
\text { psychological, emotional and social } \\
\text { distress }\end{array}$ & $\begin{array}{l}\text { Changes in length of sessions; addition of } \\
\text { orientation sessions; transition to online } \\
\text { delivery, including apps for smartphones }\end{array}$
\end{tabular}

Transition to survivorship programmes To support cancer survivors' transition from active (intensive) cancer treatment to routine follow-up care

Transition to primary care programmes

To support cancer survivors' transition from specialist-led follow-up care to primary care-led follow-up
To help cancer survivors return to a 'new normal' after cancer treatment and/or to support lifestyle changes to improve overall health and well-being
Automatic referrals to programme; changes in timing of delivery; changes in setting (cancer centre vs community); addition of content (eg, self-management)

Tailoring of tools (eg, specific recommendations, list of community resources) to cancer types; changes in delivery mode (eg, mailed vs faxed vs emailed communications)

Addition of orientation sessions; automatic registration; transition to online delivery; refinement of websites; addition of content (eg, sexuality and cancer); changes in frequency, timing and length of delivery 
develop trust and a sense of ownership with regard to the innovation. As one participant said:

... the consultations in advance and the getting the people on board and having their input into how things are gonna look and design, I think that was required in order to get any of them on board for something that would be a voluntary change in practice. (Participant 8)

Second, engagement contributed to the practice-based adaptation of the innovations to optimise fit to the local setting. This engagement occurred through mechanisms such as establishing steering or advisory committees composed of key stakeholders, conducting needs assessments, meeting with multidisciplinary cancer site teams/ tumour boards, consulting with primary care providers/ networks and codesigning with patient and/or physician groups. Participants described engagement as positively changing both the engaged person (through building a sense of ownership and personal investment; discussed below) and the innovation itself (through adaptation to the local setting; discussed above), both viewed as essential to sustainability.

\section{Ongoing education and training}

Participants across all organisations and jurisdictions emphasised that ongoing education and training was required to sustain their innovations. This was particularly true due to high staff turnover, which was deemed prevalent across organisations and jurisdictions. The nature of academic healthcare settings, with turnover of learners on an ongoing and frequent basis, was also described as a challenge to sustainability:

Probably one of the biggest barriers is that there's always new staff that come along, like fellows and residents and stuff like that. So, um, you know, they're often just not even aware. So unless there's some kind of process in place to sort of orient them to those types of things then they won't be delivering it.

(Participant 16)

Ongoing training was viewed as particularly important in cancer survivorship care given the absence of formal education and training in survivorship issues for most healthcare providers. One participant put it this way: '.. teaching, teaching the next one, so that ... we can grow our force of people involved in survivorship care. If we don't invest in that then, well then, I'm never gonna have a vacation' (Participant 24).

\section{Necessary implementation outcome}

Widespread staff and organisational buy-in

Participants stated that ongoing and widespread staff and organisational buy-in is absolutely necessary for sustainability. One participant summed this up by saying, 'without buy in and support from the physicians, from other nurses, from the other allied health staff, from the receptionist, from everybody, um, the program wouldn't work' (Participant 23). Participants discussed many factors during the implementation period that lead to widespread buy-in, including attributes of the innovation itself (eg, ease of use), how the innovation is initially framed/presented (including its evidence base), stakeholder engagement and whether it is a priority of senior management. Participants also stated that the overall low priority of survivorship care (relevant to other areas of cancer care) serves to decrease buy-in from all levels of the organisations.

\section{Important but not necessary}

The data suggested nine factors were important to sustainability, but not necessary. These are presented in table 2 . For example, while resources in the form of funding, equipment and physical space are very important for many innovations, the data demonstrated that, with the exception of one innovation, the loss of the initial implementation funding, or the lack of additional funding, equipment or space to expand beyond the implementation phase, did not result in a loss of the innovation if other necessary factors were present (eg, key people). Similarly, penetration of the innovation into existing workflows and systems, particularly hospital information technology systems, was perceived as important to many of the innovations, yet many innovations were sustained (oftentimes described as continuation of programme components and activities) in the absence of penetration when other necessary components were in place.

\section{DISCUSSION}

We investigated the factors influencing the sustainability of 25 different types of innovations in cancer survivorship care. The findings revealed a number of factors deemed necessary for sustainability: management support; organisational and system-level priorities; key people and expertise; innovation adaptation; stakeholder engagement; ongoing education and training; and staff and organisational buy-in. These findings are important given the considerable investment over the past decade to implement and scale beneficial innovations within and across Canadian jurisdictions so more people have access to high-quality cancer survivorship care. They point to specific factors implementation teams should consider and plan for to achieve their desired outcomes and maximise the long-term impact of these investments. From a practice perspective, they can be used to develop and/or select instruments and tools to assess capacity for sustainability, increase capacity in specific domains and to assist with the ongoing monitoring of key determinants and processes.

Many of the determinants, processes and outcomes identified in this study align with the emerging literature in this area. A recently developed framework ${ }^{26}$ from a 2018 review on sustainability identified four key processes that the evidence suggests are important to sustainability: partnership/engagement, training/supervision, programme 
evaluation and adaptation. Moreover, programme champions, leadership/support, resources/funding and staffing/turnover were all identified as key inner (organisational) contextual factors that influence sustainability. While the concepts may be phrased differently, our findings markedly align with the existing evidence in this area. There were also several factors identified by participants in this study that are somewhat unique, or not explicitly specified, in the literature. One of these is the speed of implementation, which participants viewed as being important because a slow(er) implementation allows implementation teams the time to plan for and implement in a way that leverages the key elements needed for sustainability. Moreover, we categorised a number of our findings as implementation outcomes, which are necessary for or important to sustainability. While we recognise that sustainability has been described as an implementation outcome itself, ${ }^{38}$ few researchers have attempted to describe or delineate the impact of more proximal implementation outcomes (eg, adoption) on the sustained use of an innovation, yet these proximal outcomes may act as moderators to sustainability (eg, continuation of programme components/activities and/or continuation of desired outcomes). Such relationships could be tested in future research.

The emphasis on the essential role of adaptation for sustainability deserves discussion. Participants were implementation leaders and relevant staff involved in the implementation and/or sustainment of innovations relevant to cancer survivorship care. Their perspectives were largely practice based, with an acute recognition that onesize-fits-all innovations do not work for most settings. This is supported by the increasing awareness in the literature that adaptation is common and likely necessary to facilitate sustainability. ${ }^{19} 263940$ In fact, the findings align well with the DSF and its postulation that innovations should not be optimised prior to implementation but rather require (and benefit from) ongoing adaptation and optimisation. In this study, innovations were adapted (eg, components, practitioners, delivery platforms) in response to changes in the practice setting (eg, staffing, information systems, processes for training) and the broader ecological system (eg, other practice settings, policies, population characteristics). It is important to note, however, that many of the described adaptations were made in response to unanticipated changes and challenges, and thus might be better termed modifications. ${ }^{39}$ These findings reinforce the need for ongoing monitoring and feedback mechanisms to assess the innovation itself and related outcomes, and changes in the setting and system at large to support appropriate and timely adaptation.

It is also important to highlight that many of adaptations described by participants were to form, rather than function. ${ }^{41}{ }^{42}$ That is, adaptations were made to specific strategies or activities (forms) rather than the intended purposes the innovation aims to achieve (functions). For example, educating and supporting patients to more effectively manage their post-treatment health concerns (function) may be accomplished through various activities, such as one-on-one teaching, individualised care plans, and so on (forms). These types of adaptations demonstrate the importance of 'flexibility within fidelity ${ }^{43}$ or fidelity-consistent adaptations ${ }^{39}$ for sustainability. In its methodology standards for studies of complex interventions, the Patient-Centered Outcomes Research Institute in the USA advises researchers and implementation teams to clearly delineate an intervention's core functions and forms, and to maintain fidelity to the core functions while documenting adaptations to form. ${ }^{44}$ By doing so, we can provide better guidance to those who implement and evaluate such interventions. This also reinforces the need for ongoing evaluation post implementation to understand the what and why of adaptations, and how these relate to sustainability; in this study, less than half of the innovations were evaluated post implementation.

We categorised widespread buy-in as an implementation outcome. We defined widespread buy-in as a commitment to the innovation by a larger group of individuals within the organisation or the organisation as a whole, specifically their commitment to support and engage in an initiative. Although we could find no clear definition or operational specificity of this concept in the existing health literature, the management and business literature does characterise buy-in in terms of one's intellectual and emotional commitment to an organisation's cause and/or plan, ${ }^{45}$ and provides guidance to increase buy-in during organisational change initiatives. ${ }^{46}$ While buy-in, as we have defined, can be present during the decision to adopt an innovation and/or its implementation, it is also a desired result of the strategies and activities (eg, communications, education/training, use of opinion leaders) put in place during implementation. By and large, implementation teams are seeking to achieve buy-in. In this way, it aligns with the definition of implementation outcomes proposed by Proctor et al, namely 'the effects of the deliberate and purposive actions to implement new treatments, practices, and services'. ${ }^{38}$ However, buy-in is not one of the eight commonly used implementation outcomes. ${ }^{38}$ We posit that perhaps this construct might be a useful addition to implementation outcomes and thereby efforts to understand how to appropriately operationalise and measure buy-in are needed.

Clearly, many of the factors presented here relate to one another and are not independent influences on sustainability. For example, stakeholder engagement (a process) often serves to increase widespread buy-in (an outcome), which then may result in additional resources (a determinant) to sustain an innovation. Moreover, both managerial support and organisational and system-level priorities (necessary determinants) will often reflect the magnitude and nature of resources (important determinant) dedicated to any initiative. Such interdependence will be present in the sustainability of any complex innovation, and demonstrates the 'messiness' of both the science and practice in this area. Future research should 
attempt to delineate what combination of factors might be most important for different types of innovations.

Several of the study findings also highlight an important issue in cancer survivorship care and programming: namely that the evidence base for survivorship innovations is of lower quality, and the resulting outcomes are 'softer', compared with the evidence base and outcomes in other areas of cancer care (eg, diagnosis and treatment). While participants in this study stated that evidence of an innovation's effectiveness contributed to sustainability by strengthening the case for funding and helping consolidate buy-in from front-line staff (see table 2), they also noted that it can be difficult to secure management support for innovations in survivorship care, in part because it does not result in quantifiable metrics like other areas of cancer care. As such, they perceived that survivorship care is viewed as a desirable, but non-essential, service within cancer care organisations. These issues undoubtedly impact the ability to sustain survivorship innovations in practice, particularly where leaders and managers have to decide between funding/resourcing services with hard performance metrics to demonstrate effectiveness versus services with metrics that are less traditional or more difficulty to quantify.

From a methodological standpoint, during sampling, we attempted to identify and categorise programmes based on Scheirer's suggested innovation types: innovations implemented by individual providers; interventions requiring coordination among multiple staff; new policies, procedures and technologies; capacity or infrastructure building; collaborative partnerships or coalitions; and broad-scale system change. ${ }^{33}$ In practice, this was challenging for several reasons. One, there are few innovations in cancer survivorship that are implemented by individual providers. Two, many of the innovations crossed categories. For example, many innovations required coordination across multiple staff, represented new policies, procedures or technologies, and involved collaborative partnerships with community-based or research-based groups. Thus, the most appropriate category was difficult to select and we therefore categorised the innovations by function (ie, its intended purpose) rather than the level or nature of the change. These are not limitations of Scheirer's taxonomy, but demonstrate the complexity that can arise when attempting to fit into pre-existing categories. We continue to advocate for and support the use of existing nomenclature, taxonomies and frameworks to help build an evidence base in this area. Nonetheless, while such frameworks should guide our work, we cannot be constrained by them when they are not helpful in the context of a particular study.

This study has a number of strengths. First, we interviewed participants from 25 different survivorship innovations across six jurisdictions, which should increase the transferability of findings. Second, we built on others' work in sustainability, including existing taxonomies and frameworks, to advance knowledge in this area. This study also has several limitations. First, this study focused solely on innovations in cancer survivorship. This may limit transferability to innovations in other areas of care, although there is no inherent reason why innovations in cancer survivorship should differ from innovations in other areas of chronic disease management that aim to address the physical, psychosocial and economic sequelae of an illness and its treatment. Given that the findings also align with the emerging literature on sustainability, conducted across a range of health conditions and settings, the findings are likely transferable. Second, although we undertook a multistepped approach to identify innovations that had been implemented across Canada, we cannot be certain that we did not miss innovations that would have been important to study. Third, we used the National Cancer Institute for Research-Tested Intervention Programs criterion for determining whether an innovation was evidence based. This criterion is not stringent and it is likely some of the innovations studied were more 'evidence-based' than others, which may have implications for sustainability (see table 2). Fourth, we attempted to discriminate between factors that are more salient or perceived by participants as necessary to sustainability as opposed to factors that are important, but not necessary. This dichotomisation may be somewhat artificial and not true for all settings or innovations. We did this in an attempt to avoid a 'laundry list' of every possible determinant of sustainability. It also attempts to address one of the gaps in our understanding of the factors that influence sustainability: namely, are some factors more critical than others? ${ }^{26}$ This is a first step toward identifying critical factors (determinants, processes and implementation outcomes) of sustainability. Future research should also focus on developing metrics and methods to prioritise these factors, and combinations thereof, and link them to appropriate strategies.

In conclusion, this study demonstrated that certain determinants, processes and implementation outcomes influence the sustainability of innovations in cancer survivorship care. These factors exist across multiple levels of the health system and are often interdependent. They also demonstrate the dynamic nature of sustainability. Three examples of this dynamism are the ongoing nature of adaptation, the shifting nature of priorities that can change the local landscape and resulting support for sustainment, and the turnover of champions and support staff. The findings may be used by researchers, decisionmakers and implementation teams to plan for sustainability during the early implementation of innovations, particularly factors shown to be necessary to the longterm use of innovations.

\section{Author affiliations}

${ }^{1}$ Community Health and Epidemiology, Dalhousie University Faculty of Medicine, Halifax, Nova Scotia, Canada

${ }^{2}$ Surgery, Dalhousie University Faculty of Medicine, Halifax, Nova Scotia, Canada ${ }^{3}$ Dalla Lana School of Public Health, University of Toronto, Toronto, Ontario, Canada ${ }^{4}$ Surgery, Nova Scotia Health Authority, Halifax, Nova Scotia, Canada

${ }^{5}$ Department of Family Practice, The University of British Columbia Faculty of Medicine, Kelowna, British Columbia, Canada 
${ }^{6}$ Brown School, Washington University in St Louis, St Louis, Missouri, USA ${ }^{7}$ Information Management \& Decision Support, Interior Health Authority, Kelowna, British Columbia, Canada

${ }^{8}$ Health Promotion, Chronic Disease and Injury Prevention, Public Health Ontario, Toronto, Ontario, Canada

${ }^{9}$ Department of Supportive Care, Princess Margaret Hospital Cancer Centre, Toronto, Ontario, Canada

\section{Twitter Sarah A Richmond @S_A_Richmond}

Acknowledgements We gratefully acknowledge the study participants who took the time to participate in this study as well as Margaret Jorgensen for her assistance with study coordination. We also acknowledge Designs that Cell for illustrating figure 1.

Contributors Conceptualisation: RU, CK, EC, BJP, GK, SAR and JLB. Methodology: RU, LLM, CK, EC, BJP, GK, SAR and JLB. Formal analysis: RU, LLM. Writing-original draft preparation: RU. Writing-review and editing: RU, LLM, CK, EC, BJP, GK, SAR and JLB. Funding acquisition: RU, CK, EC, BJP, GK, SAR and JLB. All authors read and approved the final manuscript.

Funding The work was supported by a research grant from the Canadian Cancer Society Research Institute (grant number 704897). BJP was supported in part by the US National Institute of Mental Health (K01MH113806; BJP, PI) and the US National Cancer Institute (P50CA244431; Brownson, PI).

Competing interests None declared.

Patient consent for publication Not required.

Ethics approval This study was approved by the Nova Scotia Health Authority's Research Ethics Board. All participants provided written informed consent before participating in the study.

Provenance and peer review Not commissioned; externally peer reviewed.

Data availability statement No data are available. This is a qualitative data set and therefore not publicly available due to confidentiality reasons.

Supplemental material This content has been supplied by the author(s). It has not been vetted by BMJ Publishing Group Limited (BMJ) and may not have been peer-reviewed. Any opinions or recommendations discussed are solely those of the author(s) and are not endorsed by BMJ. BMJ disclaims all liability and responsibility arising from any reliance placed on the content. Where the content includes any translated material, BMJ does not warrant the accuracy and reliability of the translations (including but not limited to local regulations, clinical guidelines, terminology, drug names and drug dosages), and is not responsible for any error and/or omissions arising from translation and adaptation or otherwise.

Open access This is an open access article distributed in accordance with the Creative Commons Attribution Non Commercial (CC BY-NC 4.0) license, which permits others to distribute, remix, adapt, build upon this work non-commercially, and license their derivative works on different terms, provided the original work is properly cited, appropriate credit is given, any changes made indicated, and the use is non-commercial. See: http://creativecommons.org/licenses/by-nc/4.0/.

ORCID iDs

Robin Urquhart http://orcid.org/0000-0001-8864-5716

Sarah A Richmond http://orcid.org/0000-0003-3632-5037

\section{REFERENCES}

1 Corkum M, Urquhart R, Kephart G, et al. Breast and cervical cancer screening behaviours among colorectal cancer survivors in nova Scotia. Curr Oncol 2014;21:670-7.

2 Johnson PM, Malatjalian D, Porter GA. Adequacy of nodal harvest in colorectal cancer: a consecutive cohort study. J Gastrointest Surg 2002;6:883-90.

3 Porter GA, Urquhart R, Bu J, et al. The impact of audit and feedback on nodal harvest in colorectal cancer. BMC Cancer 2011;11:2.

4 Rayson D, Urquhart R, Cox M, et al. Adherence to clinical practice guidelines for adjuvant chemotherapy for colorectal cancer in a Canadian Province: a population-based analysis. J Oncol Pract 2012;8:253-9.

5 Richardson DP, Porter GA, Johnson PM. Population-Based use of sphincter-preserving surgery in patients with rectal cancer: is there room for improvement? Dis Colon Rectum 2013;56:704-10.
6 Urquhart R, Folkes A, Porter G, et al. Population-Based longitudinal study of follow-up care for patients with colorectal cancer in nova Scotia. J Oncol Pract 2012;8:246-52.

7 Latosinsky S, Fradette K, Lix L, et al. Canadian breast cancer guidelines: have they made a difference? CMAJ 2007;176:771-6.

8 Cree M, Tonita J, Turner D, et al. Comparison of treatment received versus long-standing guidelines for stage III colon and stage II/ III rectal cancer patients diagnosed in Alberta, Saskatchewan, and Manitoba in 2004. Clin Colorectal Cancer 2009;8:141-5.

9 Canadian Strategy for Cancer Control. The Canadian Strategy for Cancer Control: a cancer plan for Canada - Discussion Paper. Ottawa, ON CSCC Governing Council; 2006.

10 World Health Organization. Bridging the "know-do" gap. Meeting on knowledge translation in global health. Geneva, Switzerland; 2006.

11 Ejemot RI, Ehiri JE, Meremikwu MM, et al. Hand washing for preventing diarrhoea. Cochrane Database Syst Rev 2008:CD004265.

12 Wilson KD, Kurz RS. Bridging implementation and institutionalization within organizations: proposed employment of continuous quality improvement to further dissemination. J Public Health Manag Pract 2008;14:109-16.

13 Gillissen F, Ament SM, Maessen JM. Sustainability of an enhanced recovery after surgery program (ERAS) in colonic surgery. World $\mathrm{J}$ Surg 2014

14 Kryworuchko J, Stacey D, Bai N, et al. Twelve years of clinical practice guideline development, dissemination and evaluation in Canada (1994 to 2005). Implement Sci 2009;4:49.

15 Grunfeld E. Canadian breast cancer guidelines are as effective as possible under the circumstances. CMAJ 2007;176:785-6.

16 Sheldon TA, Cullum N, Dawson D, et al. What's the evidence that NICE guidance has been implemented? Results from a national evaluation using time series analysis, audit of patients' notes, and interviews. BMJ2004;329:999.

17 Proctor E, Luke D, Calhoun A, et al. Sustainability of evidencebased healthcare: research agenda, methodological advances, and infrastructure support. Implement Sci 2015;10:88.

18 Maher L, DH G, Evans A. Sustainablity model and guide. Coventry, UK Institute for Innovation and Improvement, National Health Service; 2010. http://www.institute.nhs.uk/sustainability;

19 Chambers DA, Glasgow RE, Stange KC. The dynamic sustainability framework: addressing the paradox of sustainment amid ongoing change. Implement Sci 2013;8:117.

20 Schell SF, Luke DA, Schooley MW, et al. Public health program capacity for sustainability: a new framework. Implement Sci 2013;8:15.

21 Shelton RC, Chambers DA, Glasgow RE. An extension of RE-AIM to enhance sustainability: addressing dynamic context and promoting health equity over time. Front Public Health 2020;8:134.

22 Lennox L, Maher L, Reed J. Navigating the sustainability landscape: a systematic review of sustainability approaches in healthcare. Implement Sci 2018;13:27.

23 Moore JE, Mascarenhas A, Bain J, et al. Developing a comprehensive definition of sustainability. Implement Sci 2017;12:110.

24 Wiltsey Stirman S, Kimberly J, Cook N, et al. The sustainability of new programs and innovations: a review of the empirical literature and recommendations for future research. Implement Sci 2012;7:17.

25 Scheirer MA. Is sustainability possible? A review and commentary on empirical studies of program sustainability. Am J Eval 2005;26:320-47.

26 Shelton RC, Cooper BR, Stirman SW. The sustainability of evidencebased interventions and practices in public health and health care. Annu Rev Public Health 2018;39:55-76.

27 Canadian Cancer Society's Advisory Committee. Canadian cancer statistics 2019. Toronto, OM Canadian Cancer Society; 2019.

28 . Canadian cancer research alliance: Pan-Canadian framework for cancer survivorship research. Toronto, ON CCRA; 2017.

29 Strauss AL, Corbin JM. Basics of qualitative research: grounded theory procedures and techniques. Thousand Oaks, CA: Sage, 1990.

30 Klein KJ, Sorra JS. The challenge of innovation implementation. AMR 1996;21:1055-80.

31 Research-Tested intervention programs (RTIPs). Available: https:// rtips.cancer.gov/rtips/index.do [Accessed 4 May 2020].

32 Scheirer MA, Dearing JW. An agenda for research on the sustainability of public health programs. Am J Public Health 2011;101:2059-67.

33 Scheirer MA. Linking sustainability research to intervention types. Am J Public Health 2013;103:e73-80.

34 Nilsen P. Making sense of implementation theories, models and frameworks. Implement Sci 2015;10:53.

35 Guest G, Bunce A, Johnson L. How many interviews are enough? Field methods 2006;18:59-82. 
36 Patton MQ. Qualitative research \& evaluation methods. 3rd edn. Thousand Oaks, CA: SAGE Publications, 2002.

37 Rubin H. Rubin l: qualitative interviewing: the art of hearing data. Thousand Oaks, CA: Sage Publications, 1995.

38 Proctor E, Silmere H, Raghavan R, et al. Outcomes for implementation research: conceptual distinctions, measurement challenges, and research agenda. Adm Policy Ment Health 2011;38:65-76.

39 Wiltsey Stirman S, Baumann AA, Miller CJ. The FRAME: an expanded framework for reporting adaptations and modifications to evidence-based interventions. Implement Sci 2019;14:58.

40 Aarons GA, Green AE, Palinkas LA, et al. Dynamic adaptation process to implement an evidence-based child maltreatment intervention. Implement Sci 2012;7:32.

41 Hawe P, Shiell A, Riley T. Complex interventions: how "out of control" can a randomised controlled trial be? BMJ 2004;328:1561-3.

42 Jolles MP, Lengnick-Hall R, Mittman BS. Correction to: core functions and forms of complex health interventions: a patient- centered medical home illustration. J Gen Intern Med 2019. doi:10.1007/s11606-019-04885-z. [Epub ahead of print: 01 Apr 2019].

43 Kendall PC, Beidas RS. Smoothing the TRAIL for dissemination of evidence-based practices for youth: flexibility within fidelity. Prof Psychol 2007;38:13-20.

44 PCORI methodology standards for studies of complex interventions. Available: https://www.pcori.org/research-results/about-ourresearch/research-methodology/pcori-methodology-standards Complex [Accessed 4 May 2020].

45 Thomson K, de Chernatony L, Arganbright L, et al. The Buy-in benchmark: how staff understanding and commitment impact brand and business performance. Journal of Marketing Management 1999:15:819-35.

46 Kotter JP. Leading change: why transformation efforts fail Harvard Business Review; 1955: 59-67. 